\title{
Correlation of the Tokamak H-Mode Density Limit with Ballooning Stability at the Separatrix
}

\author{
T. Eich ${ }^{1}$, R.J. Goldston ${ }^{2}$, A. Kallenbach ${ }^{1}$, B. Sieglin ${ }^{1}$, \\ H.J. Sun ${ }^{1}$, ASDEX Upgrade Team ${ }^{1}$, JET Contributors $\ddagger$ \\ ${ }^{1}$ Max-Planck-Institut für Plasmaphysik, Boltzmannstraße 2, D-85748 Garching, \\ Germany \\ ${ }^{2}$ Princeton Plasma Physics Laboratory, Princeton, NJ 08543, USA \\ E-mail: thomas.eich@ipp.mpg.de
}

\begin{abstract}
We show for JET and ASDEX Upgrade, based on Thomson-scattering measurements, a clear correlation of the density limit of the tokamak H-Mode highconfinement regime with the approach to the ideal ballooning instability threshold at the periphery of the plasma. It is shown that the MHD ballooning parameter at the separatrix position $\alpha_{\text {sep }}$ increases about linearly with the separatrix density normalized to Greenwald density, $n_{e, s e p} / n_{G W}$ for a wide range of discharge parameters in both devices. The observed operational space is found to reach at maximum $n_{e, s e p} / n_{G W} \approx$ 0.4-0.5 at values for $\alpha_{\text {sep }} \approx 2-2.5$, in the range of theoretical predictions for ballooning instability. This work supports the hypothesis that the H-Mode density limit may be set by ballooning stability at the separatrix.
\end{abstract}

PACS numbers: 52.55.Fa

$\ddagger$ See author list of X. Litaudon et al., 2017 Nucl. Fusion 57102001 


\section{Introduction}

Operation of tokamaks with H-Mode [1] characteristics and at high densities is generally foreseen for future fusion power plants [2]. Here we show for JET and ASDEX Upgrade H-Mode plasmas, based on Thomson-scattering measurements, a clear correlation of the density limit of the tokamak H-Mode high-confinement regime with the approach to the ideal ballooning instability threshold at the periphery of the plasma. Based on the prediction of the tokamak scrape-off layer width by an empirical scaling [3], over a wide range of magnetic field, plasma current, heating power, and machine size the measured value of the ballooning-scaled dimensionless pressure gradient, $\alpha$ (see e.g.[4]), rises about linearly with the separatrix (edge) density normalized to the Greenwald value[5], until it reaches the ideal stability limit of $\alpha \approx 2-2.5$, where confinement degrades and H-Mode characteristics are terminated. This generally occurs at about $0.8-0.9$ of the lineaveraged Greenwald density limit [5] for conventional L-Mode and Ohmically-heated plasmas $[6]$.

Fluid computations put forward by Rogers et al.[7] showed resistive ballooning is strongly unstable at high edge collisionalities and identified this already in 1998 as a possible cause of density limits in tokamak operation. Extensive experimental work by LaBombard et al. shortly thereafter established that connection via the critical role of $\alpha$ in the edge region for the ballooning instability [8]. Subsequent work by the same authors $[9,10]$ did report a clear relation between the ballooning parameter and the an edge collisionality parameter, also consistent with Rogers work. BOUT calculations by $\mathrm{Xu}$ [11] supported these observations. Further notable are observations where analysis of experimental data showed that the frequency and amplitude of blob shedding increases as the density is increased $[12,13,14,15,16]$. An increasing level of filamentary transport at high densities was also recognized in H-Mode density limit studies in ASDEX Upgrade to be correlated to a degradation of confinement and a back transition to L-Mode conditions [17].

Though the underlying physics has been well-investigated, a first principles, selfconsistent, predictive theory is not yet revealed. Our work suggests the separatrix density, normalized to the Greenwald density[5], as the central parameter for the onset of the H-L back transition and gives a quantitative description for a critical edge density, well in line with a large data base from JET and ASDEX Upgrade presented here. The idea here is to use the (empirical) scaling of the power width measured at the divertor target and to show proportionality to the SOL scale lengths at the separatrix position. This strategy was first outlined, though with some more coarse assumptions, by applying the heuristic drift-based model[18]. Our finding could constitute an important advance for further studies and in practise operating tokamaks at high density. This may help to improve the economic practicality of fusion power production. This becomes clear when recalling that in a nuclear fusion power plant, a moderate increase of $10 \%$ in the core density would result in about 30\% increase of the net electrical power output [19]. Of similar importance is the access to detached plasma conditions to meet the 


\begin{tabular}{cccccc} 
& $\mathrm{I}_{p} / \mathrm{MA}$ & $\mathrm{B}_{\text {tor }} / T$ & $\mathrm{q}_{\text {cyl }}$ & $\mathrm{P}_{\text {sep }} / \mathrm{MW}$ & $f_{G W}$ \\
\hline JET-ILW & $1.0-2.5$ & $1.0-2.5$ & $2.6-4.4$ & $2-15$ & $0.5-0.9$ \\
JET-C & $1.5-3.5$ & $1.5-2.8$ & $2.4-3.2$ & $3-12$ & $0.7-0.9$ \\
\hline W-AUG & $0.6-1.2$ & $1.8-2.5$ & $2.5-6.2$ & $1-14$ & $0.4-0.9$ \\
+Pellets & 1.04 & 2.5 & 3.3 & 5 & $1.0-1.3$ \\
$\# 34613$ & 0.82 & 2.5 & 4.5 & 9 & $0.7-1.0$ \\
$\# 34971$ & 0.82 & 2.0 & 3.9 & 5 & $0.6-0.9$ \\
\hline
\end{tabular}

Table 1. Discharge parameters of analysed plasmas.

requirements of power exhaust, which scales strongly with the achievable density at the separatrix position. An increase of the separatrix density in a reactor would decrease quadratically the necessary impurity concentration to reach high scrape-off-layer (SOL) radiation fraction and hence further enlarge its operational range [20, 21, 22].

\section{Experimental methodology}

Our data base contains plasma discharges from ASDEX Upgrade (AUG) with fullW plasma facing components. The JET data base is a subset of the data that were used for the empirical scaling work for the power width with carbon walls (JETC) [3], their comparison discharges in ITER-like wall (JET-ILW) [23] and for which Thomson Scattering data in the SOL during inter-ELM periods is of sufficient quality for determining the SOL $T_{e}$ and $n_{e}$ decay length. All plasmas consist of deuterium as the main ion species and have, except where noted, H-Mode confinement factors of $\mathrm{H} 98_{y, 2} \geq 0.75$. The data base covers a large operational range for both machines and is summarized in Table 1.

The ballooning parameter for the separatrix position, $\alpha_{\text {sep }}$, is written as

$$
\alpha_{\text {sep }}=\frac{R q_{c y l}^{2}}{B_{\text {tor }}^{2} / 2 \mu_{0}} \cdot \frac{p_{\text {sep }}}{\left\langle\lambda_{p}\right\rangle}
$$

with $\mu_{0}$ the magnetic vacuum permeability, $p_{\text {sep }}$ the plasma pressure and $\left\langle\lambda_{p}\right\rangle$ the poloidal averaged pressure decay length at the separatrix position. The toroidal magnetic field is denoted as $B_{t o r}$, the cylindrical safety factor as $q_{c y l}$, the plasma current as $I_{p}$ and the major radius as $R\left(R_{J E T}=2.95 \mathrm{~m}, R_{A U G}=1.65 \mathrm{~m}\right)$. The power crossing the separatrix, $P_{s e p}$, is calculated by subtracting the radiated power from the heating power. The Greenwald density is defined as $n_{G W}=10^{14} \cdot \frac{I_{p}}{\pi a^{2}}\left[\mathrm{~m}^{-3}\right]$ [6] and the Greenwald density fraction as $f_{G W}=n_{a v} . / n_{G W}$ with $n_{a v}$. being the line averaged density.

Earlier studies using Thomson Scattering data in ASDEX Upgrade [24] show a correlation between the density and temperature decay lengths at the separatrix position $\lambda_{T} \approx \frac{2}{3} \cdot \lambda_{n}[24]$. It was also shown that $\lambda_{T} \approx \frac{7}{2} \cdot \lambda_{q}$, with $\lambda_{q}$ being the power fall-off length, by comparing outer mid plane and divertor scaling [3]. The pressure decay length is given by $\lambda_{p}^{-1}=\lambda_{T}^{-1}+\lambda_{n}^{-1}$. The ratios between the various decay lengths are 
thus

$$
\lambda_{q} \approx \frac{2}{7} \lambda_{T} \approx \frac{4}{21} \lambda_{n} \approx \frac{1}{2} \lambda_{p}
$$

In Fig.1 an example of temperature and density fall-off estimation for each device is shown. The results for the JET data base are $\lambda_{p} / \lambda_{q} \sim 2.1, \lambda_{T} / \lambda_{q} \sim 3.7, \lambda_{n} / \lambda_{T} \sim 1.5$, and hence well in line with the measurements at AUG. Figure 2 shows the ratio between $\lambda_{p}$ (from Thomson Scattering) and $\lambda_{q}$ (from the power width scaling[3]) versus the separatrix density normalized by the Greenwald density. It should be noted that $\lambda_{p} / \lambda_{q}$ shows no correlation with the normalized separatrix density.

To derive the separatrix pressure, $p_{\text {sep }}$ and the pressure fall-off length, $\lambda_{p}$ at the mid plane we use the same method as in AUG [24]. However, note that for JET no magnetic mapping was applied as the Thomson diagnostic in JET measures at the outer mid plane and the magnetic configuration is kept constant[25]. Data are taken in between ELMs, representing 50-95\% of the ELM cycle time for about one second during flat top phase. To estimate the separatrix position we apply the model by Stangeby [26] to estimate the electron temperature, $T_{e, s e p}$, there (typically $\approx 100 \mathrm{eV}, T_{e}=T_{i}$ ).

$$
T_{e, s e p} \approx\left(\frac{7}{4} \frac{P_{\text {sep }} \cdot L_{c}}{\kappa_{0}^{e} \cdot A_{S O L}}\right)^{\frac{2}{7}}
$$

We use $a$ for the minor radius and $\kappa_{\text {geo }}$ for the plasma elongation. The connection length, $L_{c}$ from the outer mid plane region to the divertor is $L_{c}=\pi q_{c y l} R$. The effective area, $A_{S O L}$ writes as $A_{S O L}=4 \pi R\left\langle\lambda_{q}\right\rangle \cdot \frac{\left\langle B_{\text {pol }}\right\rangle}{B_{t o r}}$ with $\left\langle\lambda_{q}\right\rangle$ as the poloidally averaged power

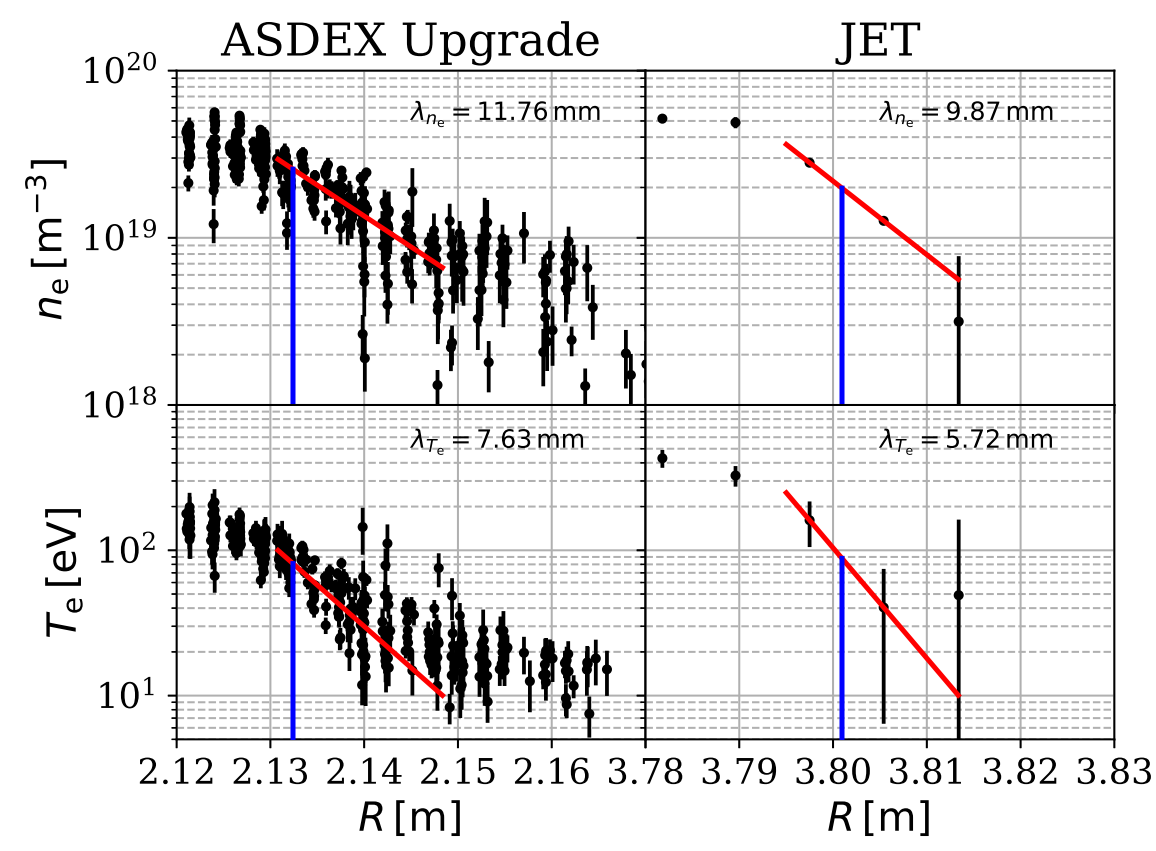

Figure 1. Mid plane profiles measured by Thomson Scattering. The separatrix location (blue) and decay lengths (red) are shown. (AUG:\#32291, $I_{p}=0.8 \mathrm{MA}$, $B_{\text {tor }}=2.5 \mathrm{~T}, P_{\text {sep }}=4 \mathrm{MW}$, JET: $\#$ 82448, $\left.I_{p}=2.0 \mathrm{MA}, B_{\text {tor }}=2.1 \mathrm{~T}, P_{\text {sep }}=5 \mathrm{MW}\right)$ 


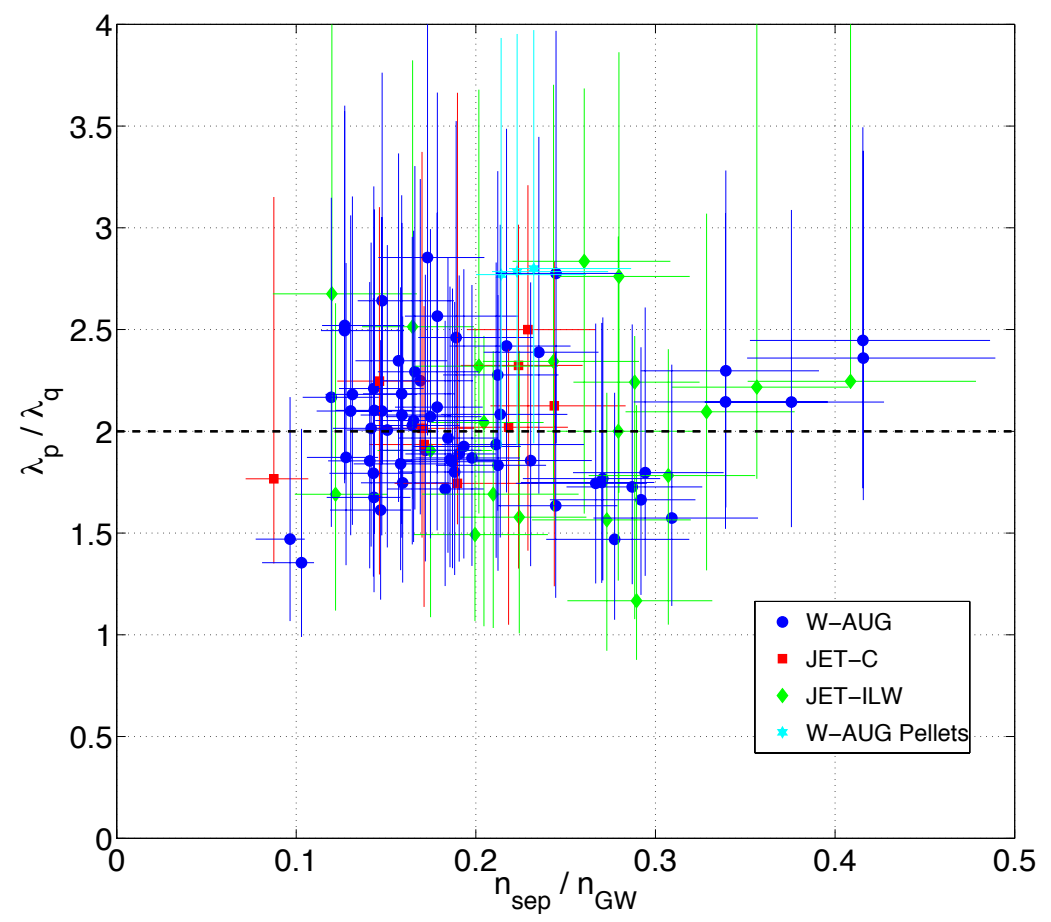

Figure 2. Ratio of the pressure and divertor heat flux decay length vs the separatrix normalized to the Greenwald density.

fall-off length. Using $\hat{\kappa}=\left(\left(1+\kappa_{\text {geo }}^{2}\right) / 2\right)^{0.5}, A=R / a, q_{c y l}=\frac{B_{t o r}}{\left\langle B_{\text {pol }}\right\rangle} \cdot \frac{\hat{\kappa}}{A},\left\langle B_{\text {pol }}\right\rangle=\frac{\mu_{0} I_{p}}{2 \pi a \hat{\kappa}}$ and $\kappa_{0}^{e}$ being the Spitzer-Härm electron heat conduction constant $\left(\kappa_{0}^{e}=2000(\mathrm{eV})^{-\frac{7}{2}} \mathrm{Wm}^{-1}\right)$ we rewrite Eq.3 as

$$
T_{e, s e p} \approx\left(\frac{7 P_{s e p} q_{c y l}^{2} A}{16 \kappa_{0}^{e} \hat{\kappa}\left\langle\lambda_{q}\right\rangle}\right)^{\frac{2}{7}}
$$

\section{Separatrix ballooning parameter}

In order to verify if the proximity to a critical value of $\alpha_{\text {sep }}$ explains the H-Mode density limit we plot in Fig.3 the separatrix density $n_{e, s e p}$ normalized by the Greenwald density, $n_{G W}$, versus the ballooning parameter, $\alpha_{\text {sep }}$. The values of $\alpha_{\text {sep }}$ are calculated by inserting the mid plane parameters $n_{e, s e p}, T_{\text {sep }}, \lambda_{n}$ and $\lambda_{T}$, assuming $T_{e}=T_{i}$. Fig.3 shows an about linear relation between $n_{e, s e p} / n_{G W}$ for $\alpha_{\text {sep }} \leq 2$. Remarkably, data from machines a factor of nearly two different in major radius, over a range of a factor of three in plasma current and magnetic field, as well as a factor of over ten in heating power fall together on this curve. In discharge \#34613 (AUG) strong gas puffing was performed, without cryopumping. Both the line averaged and separatrix density reach very high values. The back transition to the L-Mode is observed at about $n_{e, s e p} / n_{G W} \approx$ 0.5 and at $\mathrm{H}_{98 y, 2} \leq 0.75$. The back transition from H- to L-mode is correlated with a reduction of the radial electric field in the confinement region close to the separatrix, 


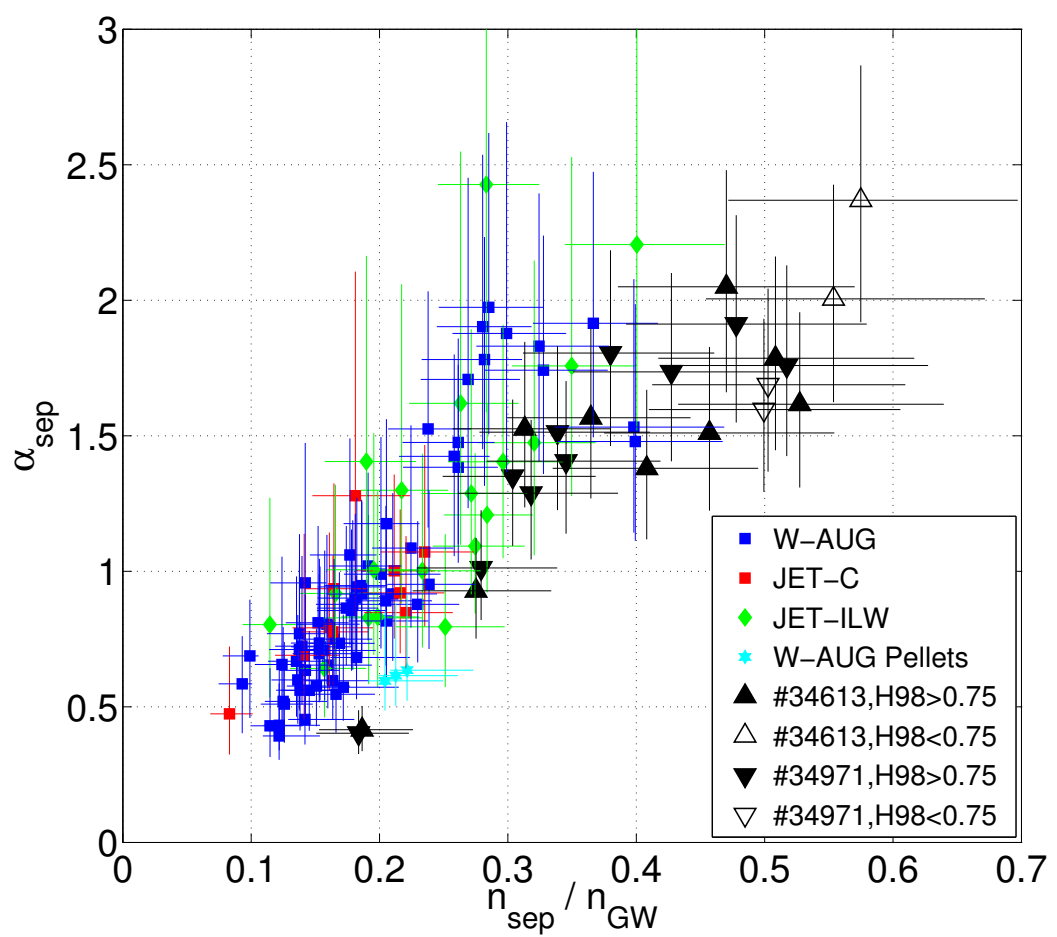

Figure 3. Ballooning parameter, $\alpha_{\text {sep }}$ calculated from Thomson data, versus the normalized separatrix density $n_{e, s e p} / n_{G W}$.

estimated by $\nabla T_{i} /(e n)$, dropping below the critical value of about $12 \mathrm{kV} / \mathrm{m}$ [27]. This possible mechanism for the $\mathrm{H}-\mathrm{L}$ back transition is well in line with the previously mentioned work of increased filamentary transport and consecutive flattening of the edge profiles $[8,9,10,11,12,13,14,15,16,17]$, since the reduction of $\nabla T_{i} /(\mathrm{en})$ leads to the reduction of the electric field in the vicinity of the separatrix.

Fig.4 displays the normalized separatrix density versus the Greenwald density fraction, $n_{a v} . / n_{G W}$. All discharges (except pellet injected) are fueled by gas puffing. The highest values for $n_{e, s e p} / n_{G W}$ are only achieved at high Greenwald density fractions and hence the H-Mode density limit is only observed at $n_{e, a v .} / n_{G W} \approx 0.8-0.9$. Pellet fueled discharges have proven that the Greenwald density fraction can exceed unity[28]. Fig.3 shows that for a pellet injected H-Mode plasma $\alpha_{\text {sep }} \approx 0.6$ and $n_{e, s e p} / n_{G W} \approx 0.2$ whilst $n_{e, a v} / n_{G W} \approx 1-1.25$ is measured not violating the H-Mode density limit mechanism proposed here. Discharge \#34613 and \#34971 presumably develop along the upper range of the data base for $n_{e, s e p} / n_{G W}$ due to the absence of pumping. In general the variation of the ratio between the separatrix and line averaged density reflects the large operational condition the data base represents. For example data from JET operation with carbon plasma-facing components are observed to reach high line averaged density and simultaneous low edge density. 


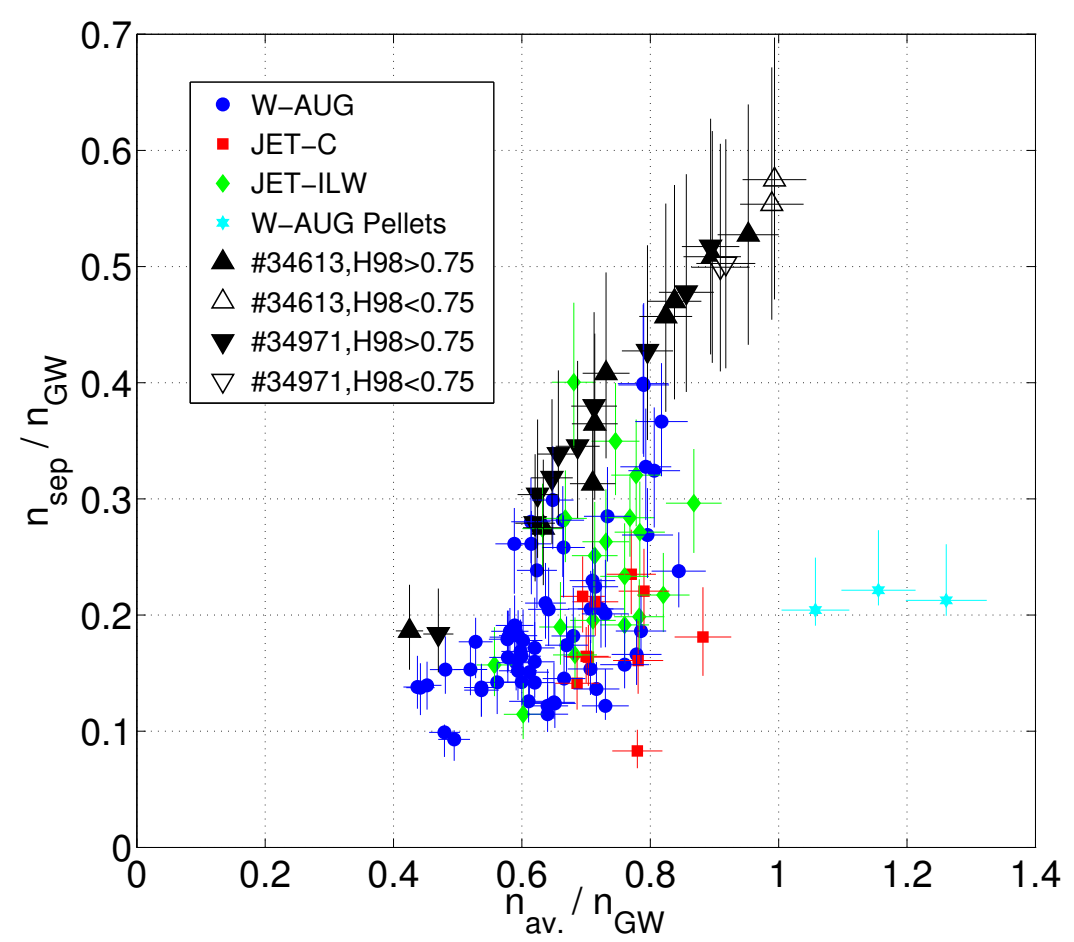

Figure 4. The normalized separatrix density, $n_{e, s e p} / n_{G W}$, versus the normalized line average density, $n_{e, a v} . / n_{G W}$.

\section{Critical separatrix density}

The concept of the ballooning limit was originally derived for closed field lines. Ricci[29] and Halpern [30] provided formal support for the extension of their turbulence models onto open field lines. The instability arises because under conditions of high collisionality when average minimum B stabilization fails - parallel dynamics cannot compensate drive from bad curvature regions through connection to good curvature.

The critical value for $\alpha$ in circular plasmas is given by $0.6 s$ [4], and the shear, $s$, in the scrape off layer should be close to 2, since the currents are small. Early local calculations indicated a linear dependence on elongation [31]. Numerical calculations of global stability by Bernard et al. [32] gave $\alpha_{\text {crit }} \approx \kappa_{\text {geo }}^{1.2}(1+1.5 \delta) \approx 2.5$ for typical triangularities in the data base of $\delta=0.2$. Mauel [33] reported $\alpha_{\text {crit }} \approx 2.2$ for $\kappa_{\text {geo }}=2$. Numerical calculations by Makowski for DIII-D, just inside the separatrix, give $\alpha_{\text {crit }} \approx 2.5[34]$.

Thus, a critical separatrix density, $n_{e, s e p}^{\text {crit }}$ is defined when the ballooning parameter divided by $\alpha_{s e p}^{c r i t}$ reaches unity. Setting $p_{\text {sep }}=2 n_{e, s e p}^{\text {crit }} T_{\text {sep }},\left(T_{e}=T_{i}=T_{\text {sep }}\right)$ gives

$$
\frac{\alpha_{\text {sep }}}{\alpha_{\text {sep }}^{\text {crit }}}=\frac{R \cdot q_{c y l}^{2} \cdot 2 \mu_{0} \cdot 2 n_{e, s e p}^{\text {crit }} T_{\text {sep }}}{\alpha_{\text {sep }}^{\text {crit }} \cdot B_{\text {tor }}^{2} \cdot\left\langle\lambda_{p}\right\rangle} \approx 1
$$


Approximating $\left\langle\lambda_{p}\right\rangle \approx 2\left\langle\lambda_{q}\right\rangle$ and $T_{\text {sep }}$ by Eq. 4 we get

$$
n_{e, s e p}^{\text {crit }} \approx \frac{\alpha_{\text {sep }}^{\text {crit }}}{2 \mu_{0}}\left(\frac{16 \kappa_{0}^{e} \hat{\kappa}}{7 A}\right)^{\frac{2}{7}} \frac{B_{\text {tor }}^{2}}{P_{\text {sep }}^{\frac{2}{7}} \cdot R \cdot q_{\text {cyl }}^{\frac{18}{7}}} \cdot\left\langle\lambda_{q}\right\rangle^{\frac{9}{7}}
$$

An accurate empirical scaling for JET and AUG H-Mode plasmas for $\lambda_{q}$ at the outer mid plane is

$$
\lambda_{q}[\mathrm{~mm}]=0.73 B_{\text {tor }}^{-0.78} q_{\text {cyl }}^{1.2} P_{\text {sep }}^{0.1} R^{0.02}
$$

with $P_{\text {sep }}$ in units of $[\mathrm{MW}]$ and $\lambda_{q}$ in [mm] [3]. We rewrite the scaling using [W] and $[\mathrm{m}]$ and set the exponents for $B_{t o r}$ and $q_{c y l}$ as multiples of $1 / 9$ (Note: $(7 / 9) / 0.78=0.997$, $(11 / 9) / 1.2=1.019)$. We ignore the exponent in $R$. The power width at the outer mid plane, $\lambda_{q}$, and the poloidally averaged power width, $\left\langle\lambda_{q}\right\rangle$, are correlated as $\lambda_{q}=0.55 \pm 0.05 \cdot\left\langle\lambda_{q}\right\rangle$ for all used data here (see Eq.9 in [3]). Correspondingly we calculate $C_{\left\langle\lambda_{q}\right\rangle}=0.73 \cdot 10^{-3} \cdot(0.55)^{-1} \cdot\left(10^{6}\right)^{-0.1}=3.33 \cdot 10^{-4}$ in units of $\left[\mathrm{mT}^{7 / 9} \mathrm{~W}^{-1 / 10}\right]$ and rewrite the scaling as:

$$
\left\langle\lambda_{q}\right\rangle[m]=C_{\left\langle\lambda_{q}\right\rangle} \cdot B_{\text {tor }}^{-\frac{7}{9}} \cdot q_{c y l}^{\frac{11}{9}} \cdot P_{\text {sep }}^{\frac{1}{10}}
$$

Inserting Eq.8 into Eq.6 gives

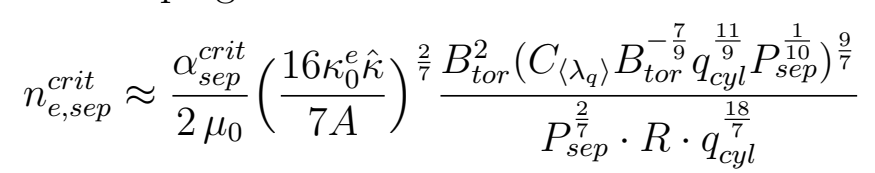

which reduces to linear terms for $B_{t o r}$ and $q_{c y l}$

$$
n_{e, s e p}^{c r i t} \approx C_{\left\langle\lambda_{q}\right\rangle}^{\frac{9}{7}} \frac{\alpha_{s e p}^{c r i t}}{2 \mu_{0}}\left(\frac{16 \kappa_{0}^{e} \hat{\kappa}}{7 A}\right)^{\frac{2}{7}} \cdot P_{s e p}^{-\frac{11}{70}} \cdot \frac{B_{t o r}}{R \cdot q_{c y l}}
$$

By identifying

$$
\frac{B_{\text {tor }}}{\mu_{0} \cdot R \cdot q_{\text {cyl }}}=\frac{I_{p}}{2 \pi \cdot a^{2} \cdot \hat{\kappa}^{2}}
$$

we rewrite Eq.10 as

$$
n_{e, s e p}^{c r i t} \approx C_{\left\langle\lambda_{q}\right\rangle}^{\frac{9}{7}} \frac{\alpha_{s e p}^{c r i t}}{4}\left(\frac{16 \kappa_{0}^{e}}{7 A}\right)^{\frac{2}{7}} \hat{\kappa}^{-\frac{12}{7}} P_{s e p}^{-\frac{11}{70}} \cdot \frac{I_{p}}{\pi a^{2}}
$$

Inserting the values for $\kappa_{0}^{e}, C_{\left\langle\lambda_{q}\right\rangle}$, the Greenwald density and expressing $\hat{\kappa}$ in terms of $\kappa_{\text {geo }}$ leads to

$$
n_{e, s e p}^{c r i t} \approx 5.9 \cdot \alpha_{s e p}^{c r i t} \cdot A^{-\frac{2}{7}}\left(\frac{1+\kappa_{g e o}^{2}}{2}\right)^{-\frac{6}{7}} P_{s e p}^{-\frac{11}{70}} \cdot n_{G W}
$$

For typical parameter in JET and AUG like $\kappa_{\text {geo }}=1.7, A=3$ and $P_{\text {sep }}=5 \cdot 10^{6} \mathrm{~W}$ this results in

$$
n_{e, s e p}^{\text {crit }} \approx 0.21 \cdot \alpha_{\text {sep }}^{\text {crit }} \cdot n_{G W}
$$

By setting $\alpha_{\text {sep }}^{c r i t}=2.0-2.5$ we find in line with the data

$$
n_{e, s e p}^{\text {crit }} \approx 0.42-0.53 \cdot n_{G W}
$$

The ion and electron temperature and fall-off lengths were assumed here to be similar, which should be reasonably well fulfilled for $n_{e, s e p} / n_{G W} \sim 0.4-0.5$. 


\section{Conclusion}

The MHD-normalized pressure gradient at the separatrix, $\alpha_{\text {sep }}$, in both ASDEX Upgrade and JET is measured, using high-resolution Thomson scattering, to rise linearly with the separatrix density normalized to the Greenwald value. These normalized data gather together into a single curve over a wide range of plasma parameters, consistent with earlier predictions based on the heuristic drift-based model[18]. When $\alpha_{\text {sep }}$ reaches $\sim 2-2.5$, consistent with the theoretically predicted onset of ballooning modes, confinement degrades and the density limit of the H-Mode is found.

Dedicated studies need to follow aiming at direct observation of ballooning or related modes at the plasma edge, for high values of $n_{e, s e p} / n_{G W}$. Our results indicate that if shallow pellet injection, as planned for ITER, increases the ratio of $n_{e, a v} / n_{e, s e p}$ compared with gas fueling, it would allow higher average normalized density operation, and so higher performance, in ITER. This should be explored experimentally on existing devices. The empirical scaling for the power width, so far, does not include shaping effects like elongation and triangularity. This topic should be addressed in dedicated heat flux studies. The critical separatrix density is found to scale (as $\alpha_{\text {crit }} \propto \kappa_{\text {geo }}$ ) with $n_{e, \text { sep }}^{\text {crit }} \propto \kappa_{\text {geo }}\left(1+\kappa_{\text {geo }}^{2}\right)^{-6 / 7}$, and hence appears to be almost independent of elongation for typical values of $\kappa_{g e o}$ in tokamaks. This finding should be challenged e.g in TCV with its large capabilities for plasma shaping. Further experiments should study the effect of high triangularity, which is observed to allow for higher Greenwald density fractions before confinement degradation is observed[35, 36], for the critical separatrix density. The power dependency on the critical separatrix density is found to be very weak, broadly consistent with the finding of Huber at JET[36] and Petrie at DIII-D[37] when comparing the line averaged density normalized to Greenwald with heating power. However, it should be noted that the $P_{\text {sep }}$ in Eq.13 represents the power crossing the separatrix in type-I inter-ELM periods, as it was used for the empirical power width scaling[3]. Further analysis is needed to extent the proposed scaling e.g. to heating power or ELM averaged power crossing the separatrix.

Generally, our suggestion for understanding of the density limit in H-mode plasma may open up the possibility of innovation to improve tokamak fusion power systems. We finally note that the possible connection between the back transition from high to low confinement conditions, known as the H-Mode density limit, and the disruptive density limit in L-Mode plasmas requires further studies.

\section{Acknowledgement}

This work has been carried out within the framework of the EUROfusion Consortium and has received funding from the Euratom research and training programme 2014-2018 under grant agreement No 633053. The views and opinions expressed herein do not necessarily reflect those of the European Commission. The work of RJG was supported by US DOE Contract No. DE-AC02-09CH11466. 


\section{References}

[1] F.Wagner, Phys. Rev. Lett. 49, 1408 (1982)

[2] H.Zohm, C.Angioni, E.Fable et al. Nucl. Fusion, Vol. 53, No. 7 (2013)

[3] T.Eich, B.Sieglin, A.Scarabosio et al., Phys. Rev. Lett. 195003 (2011)

[4] H.Zohm, Magnetohydrodynamic Stability of Tokamaks, WILEY-VCH (2015)

[5] M.Greenwald, J.L.Terry, S.M.Wolfe, Nucl. Fusion, Vol.28, No.12 (1988)

[6] M.Greenwald, Plasma Phys.Contr.Fusion,R27, 44 (2002)

[7] B. N. Rogers, J. F. Drake, and A. Zeiler, Phys. Rev. Lett. 81, 4396 (1998).

[8] B. LaBombard, R. L. Boivin, M. Greenwald et al., Phys. Plasmas 8, p.2107 (2001).

[9] B. LaBombard, J. Hughes, D. Mossessian, et al. , Nucl. Fusion 45, 1658 (2005).

[10] B. LaBombard, J. W. Hughes, N. Smick, et al. Phys. Plasmas 15, 056106 (2008).

[11] X.Q.Xu, W.M. Nevins, T.D.Rognlien et al. Phys. Plasmas 10, p.1773 (2003)

[12] V. Naulin, Phys. Plasmas 10, p.4016 (2003).

[13] O.E. Garcia, R.A. Pitts , J. Horacek et al., Plasma Phys. Control. Fusion 49, B47-57 (2007)

[14] O.E. Garcia, S.M. Fritzner, R. Kube et al., Phys. Plasmas 20, 055901 (2013)

[15] R. Kube, O. E. Garcia, B. LaBombard et al., J.Nucl. Mater., 438, S505S508 (2013)

[16] D.Carralero, G.Birkenmeier, H.W.Mller et al., Nucl. Fusion 54123005 (2014)

[17] M.Bernert, T.Eich, A.Kallenbach et al., Plasma Phys. Control. Fusion 57014038 (2015)

[18] R.J.Goldston and T.Eich, IAEA FEC, TH/P4-19 (2012)

[19] R.Wenninger, R.Kembleton, C.Bachmann et al., Nucl. Fusion, p.16011 Vol.57 (2017)

[20] A.S. Kukushkin, H.D.Pacher, G.W. Pacher et al., J. Nucl. Mater., p.S203-207, Vol. 438 (2013)

[21] M.L.Reinke, Nucl. Fusion 57 (2017) 034004

[22] R.J.Goldston, M.L.Reinke, J.A.Schwartz, Plasma Phys. Contr. Fusion, p.055015 Vol.59 (2017)

[23] B.Sieglin, T.Eich1, A.Scarabosio et al, Plasma Phys. Contr. Fusion, p.124039 Vol.55 (2013)

[24] H.J Sun, E.Wolfrum ,T.Eich et al., Plasma Phys. Contr. Fusion, p.125001 Vol.57 (2015)

[25] R. Pasqualotto et al., Rev.Sci.Inst., Vol.75, p.3891 (2004)

[26] P.C.Stangeby, The plasma boundary of magnetic fusion devices, Inst.of Phys.Publishing (2000)

[27] F.Ryter et al., Plasma Phys. Contr. Fusion, p.014007 Vol.58 (2016)

[28] P.Lang, A.Burckhardt, M.Bernert at al., Nucl. Fusion, 083009 Vol.54 (2014)

[29] P Ricci, F D Halpern, S Jolliet et al., Plasma Phys. Contr. Fusion, p.124047, Vol.54 (2012)

[30] F.Halpern, S.Jolliet, J.Loizu et al., Physiscs of Plasmas, Vol. 20, 052306 (2013)

[31] O.P. Pogutse, N.V. Chudin and E.I. Yurchenko, Sov. J. Plasma Physics 6(3) (1980) 341

[32] L.C.Bernard, F.J.Helton, R.W.Moore, T.N.Todd, Nucl. Fusion, p.1475 Vol.23 (1983)

[33] M.E.Mauel, The Physics of Fluids 30, 3843 (1987)

[34] M.A.Makowski, C.J.Lasnier, A.W.Leonard et al., Nuclear Materials and Energy (2017)

[35] G. Saibene, L.D.Horton, R.Sartori et al., Nucl. Fusion 39 p.1133 (1999)

[36] A.Huber, S.Brezinsek, G.Sergienko et al., J.Nucl.Mater., p.445-449, Vol. 463 (2015)

[37] T.W. Petrie, A.G. Kellmann, M.A. Mahdavi, Nucl. Fusion 33, p.929 (1993) 